\title{
Cathepsin L-induced galectin-1 may act as a proangiogenic factor in the metastasis of high-grade serous carcinoma
}

Md Zahidul I. Pranjol 1,2, Dmitry A. Zinovkin³, Annelie R. T. Maskell' , Laura J. Stephens',

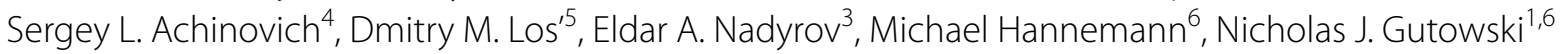
and Jacqueline L. Whatmore ${ }^{1 *}$ (D)

\begin{abstract}
Background: New treatment options for metastasised high-grade serous carcinoma (HGSC) are urgently needed. HGSC frequently metastasises to the omentum, inducing angiogenesis in the local omental microvasculature to facilitate tumour growth. We previously showed that HGSC-secreted cathepsin L (CathL) induces pro-angiogenic changes in disease relevant human omental microvascular endothelial cells (HOMECs), suggesting a role in tumour angiogenesis. Here we investigate whether CathL acts by inducing local production of the carbohydrate-binding protein galectin-1 (Gal1), which has been reported to be involved in tumourigenesis in other tumours.

Methods: HOMECs were used for all experiments. Gal1 mRNA and protein levels were measured by RT-PCR and ELISA respectively. Gal1-induced cell proliferation was assessed using WST-1 assay, migration using a transwell assay and in vivo Gal1 expression by immunohistochemistry.

Results: CathL transcriptionally regulated HOMEC production and secretion of Gal1 via activation of NFKB (significantly inhibited by sulfasalazine). Gal1 significantly enhanced HOMEC migration ( $p<0.001)$ and proliferation $(p<0.001)$, suggesting an autocrine action. The latter was significantly reduced by the MEK/ERK1/2 inhibitors U0126 and PD98059 suggesting downstream activation of this pathway. Immunohistochemical analysis of omenta from HGSC patients with or without metastatic disease demonstrated a positive correlation between Gal1 expression and number of microvessels $(r=0.8702, p<0.001)$, and area of vessels $(r=0.7283, p<0.001)$, supporting a proangiogenic role for Gal1 in omental metastases.

Conclusion: HOMEC Gal1 transcription and release in response to CathL secreted from metastasising HGSC acts in an autocrine manner on the local microvasculature to induce pro-angiogenic changes, highlighting a potential new therapeutic target.
\end{abstract}

Keywords: Galectin-1, Angiogenesis, Metastasis, High-grade serous carcinoma, Signalling pathways

\footnotetext{
*Correspondence: J.L.Whatmore@exeter.ac.uk

${ }^{1}$ Institute of Biomedical and Clinical Science, University of Exeter Medical

School, Exeter, Devon EX1 2LU, UK

Full list of author information is available at the end of the article
} 


\section{Background}

Effective treatment of patients suffering from advanced, high-grade serous carcinoma (HGSC) is still clinically challenging [1], with development of new therapies impeded by our limited understanding of the cellular mechanisms involved in HGSC metastasis.

Primary HGSC cells commonly metastasise to the omentum where secondary tumour establishment requires local neoangiogenesis to supply oxygen and nutrients. This process involves activation of a proangiogenic phenotype in the local microvascular endothelial cells (ECs), facilitated by complex cross-talk between the host omental cells and tumour cells. Growth factors and chemokines are locally secreted into the tumour microenvironment and may present potential therapeutic targets. For instance, vascular endothelial growth factor (VEGF) is highly expressed and over-secreted in HGSC [2] and thus, several anti-VEGF based therapies e.g. bevacizumab, have been developed. However, the observation that these therapies have only limited benefit in advanced disease [3-7], and that non-VEGF pathways may play a role in HGSC metastasis to the omentum has raised the possibility that alternative pro-angiogenic factors may be important e.g. cathepsins D (CathD) and L (CathL) [811]. It is thus important to understand the mechanisms by which these proteins activate angiogenic pathways in metastatic HGSC.

Our preliminary data suggested that CathL induces a differential expression of galectin1 (Gal1) mRNA (LGALS1) in disease-relevant human omental microvascular endothelial cells (HOMECs). Gal1, a carbohydratebinding protein is reported to have a range of cellular effects [12]. In cancer, increased Gal1 expression is linked to tumour progression, possibly contributing to cancer cell invasion and metastasis formation [13, 14], and is associated with increased rates of disease reoccurrence [15-19], including in HGSC [20]. Gal1 may also play a role in cancer angiogenesis since Gal1 knockout mice had severely impeded vessel formation and disrupted tumour growth [21]. This is supported by the observation that, in vitro, exogenous Gal1 induced angiogenic tube-formation in non-disease EC models such as human umbilical vein ECs (HUVECs) and EA.hy926 [22, 23]. Despite this emerging role for Gal1 in tumour angiogenesis, the molecular mechanisms involved in its cellular effects in ECs remain poorly understood.

In this investigation, we examined the role and potential cellular regulation of Gal1 in metastasis of HGSC to the omentum, using both in vitro studies on diseaserelevant HOMECs and immunohistochemical studies of patient samples. We show, for the first time that CathL, known to be secreted from HGSC, induces secretion of Gal1 from HOMECs in a transcriptionally regulated manner. Gal1 then acts in an autocrine manner to induce a proangiogenic phenotype, i.e. enhanced proliferation and migration, in these cells, via activation of the intracellular kinases ERK1/2 and AKT. Finally, we report, for the first time, an increased in vivo omental endothelial expression of Gal1 that correlates with increased microvessel density and vessel area in omentum of patients with serous carcinoma (with HGSC metastasis) compared with normal omentum or omentum of patients with non-metastatic serous carcinoma, indicating a potential proangiogenic role for Gal1 that confirms our in vitro observation. These combined data strongly indicate Gal1 as a proangiogenic factor in advanced HGSC that could be targeted therapeutically.

\section{Materials and methods}

\section{Primary HOMEC isolation and culture}

Non-malignant omental tissue samples were collected from patients undergoing surgery at the Royal Devon and Exeter NHS Foundation Trust (Exeter, United Kingdom) with ethical approval and informed written consent. HOMECs were isolated using collagen-digestion and anti-CD31 magnetic beads, characterised and cultured as primary cells as previously described [24]. Briefly, HOMECs were cultured in endothelial cell (EC) growth media (MV2, PromoCell, Heidelberg, Germany) supplemented with supplied growth factors, $5 \%(\mathrm{v} / \mathrm{v})$ foetal calf serum (FCS) and $0.1 \%(\mathrm{v} / \mathrm{v})$ gentamycin (Sigma, Poole, UK). Cells were maintained at $37{ }^{\circ} \mathrm{C}$ in a humidified atmosphere supplemented with $5 \% \mathrm{CO}_{2}$.

\section{Cell based ELISA}

Phosphorylation levels of NFKB, ERK1/2 and AKT were measured using specific cell-based ELISA kits (BioTechne Ltd., Abingdon, UK) according to the manufacturer's instructions. Cells were starved, and subsequently treated \pm recombinant human VEGF165 $(20 \mathrm{ng} / \mathrm{ml}$, positive control; Peprotech, London, UK), CathL (50 ng/ml; from human liver; Sigma-Aldrich, Poole, UK) and recombinant human Gal1 (50 ng/ml; Sigma-Aldrich, Poole, $\mathrm{UK}) \pm \mathrm{NF} \kappa \mathrm{B}, \mathrm{ERK} 1 / 2$ and $\mathrm{AKT}$ inhibitors at their given concentrations (Table 1) for $4,10 \mathrm{~min}$ or $4 \mathrm{~h}$. To confirm the effect of these inhibitors, HOMECs were pre-incubated with the inhibitors and then co-treated \pm proangiogenic factors. Fluorescence intensity was measured, and the results were expressed as fold change in phospho$\mathrm{NF} \kappa \mathrm{B}$ (p65), -ERK1/2 or -AKT relative to total NFKB, ERK or AKT levels (compared to control).

\section{Cell proliferation assay \\ WST-1 assay}

Investigation of HOMEC proliferation was as previously described [8]. Briefly, cells were seeded at a density of 
Table 1 Concentrations of treatments added

\begin{tabular}{|c|c|c|c|c|}
\hline Treatments & Purpose & Pre-incubation time & Concentration(s) & Source \\
\hline VEGF165 & Positive control & $\mathrm{n} / \mathrm{a}$ & $20 \mathrm{ng} / \mathrm{ml}$ & Peprotech (London, UK) \\
\hline $\begin{array}{l}\text { Tumour necrosis factor- } \\
\text { alpha (TNF-a) }\end{array}$ & Positive control & $\mathrm{n} / \mathrm{a}$ & $160 \mathrm{pg} / \mathrm{ml}$ & Enzo Life sciences, (Exeter, UK) \\
\hline CathL & Treatment & $\mathrm{n} / \mathrm{a}$ & $50 \mathrm{ng} / \mathrm{ml}$ & Sigma-Aldrich (Poole, UK) \\
\hline Gal1 & Treatment & $\mathrm{n} / \mathrm{a}$ & $1,5,10,25,50,125 \mathrm{ng} / \mathrm{ml}$ & Sigma-Aldrich (Poole, UK) \\
\hline Sulfasalazine & NFKB inhibitor & $24 \mathrm{~h}$ & $100 \mu \mathrm{mol} / \mathrm{l}$ & Stratech (Suffolk, UK) \\
\hline U0126 & MEK/ERK1/2 inhibitor & $20-30 \mathrm{~min}$ & $10 \mu \mathrm{mol} / /$ & Stratech (Suffolk, UK) \\
\hline PD98059 & MEKJERK1/2 inhibitor & $20-30 \mathrm{~min}$ & $25 \mu \mathrm{mol} / /$ & Stratech (Suffolk, UK) \\
\hline LY294002 & PI3K inhibitor & $1-2 h$ & $25 \mu \mathrm{mol} / /$ & Stratech (Suffolk, UK) \\
\hline MK2206 & AKT inhibitor & $1-2 \mathrm{~h}$ & $5 \mu \mathrm{mol} / \mathrm{l}$ & Stratech (Suffolk, UK) \\
\hline
\end{tabular}

$\mathrm{n} / \mathrm{a}$, Not applicable

$1 \times 10^{4}$ cells per well in $2 \%(\mathrm{w} / \mathrm{v})$ gelatin (Sigma, Poole, UK) coated 96-well plates (Greiner Bio One, Stonehouse, UK) and treated overnight in growth factor-deprived media containing $2 \%(\mathrm{v} / \mathrm{v})$ FCS. After $24 \mathrm{~h}$, treatments $(1,5,10,25,50$ and $125 \mathrm{ng} / \mathrm{ml}$ of Gall1 \pm inhibitors $)$ were added at the given concentrations (Table 1) and incubated for 48 or $72 \mathrm{~h}$. Subsequently, WST-1 reagent (Roche, Welwyn Garden City, UK) was added to the assay medium and absorbance was measured at $450 \mathrm{~nm}$ against the blank in a PHERAstar BMG plate-reader.

\section{BrdU assay}

Cells were seeded in $2 \%$ gelatin pre-coated 96 well plates at a density of 20,000 cells/well in starvation media containing 2\% FCS. After overnight incubation, cells were treated with or without Gal1 $(1,5,25,50$ and $125 \mathrm{ng} / \mathrm{ml})$ and incubated for $48 \mathrm{~h}$. A commercially available BrdU reagent (Merck Chemicals Ltd., Nottingham, UK) was added to the wells for the last 24-h incubation and cellular proliferation was assessed (according to the manufacturer's instructions) based on absorbance using a SpectraMax plate-reader (Molecular Devices, Berkshire, UK) set at dual wavelength of 450/550 nm.

\section{CyQUANT assay}

This procedure was performed according the manufacturers instruction. Briefly, after $48 \mathrm{~h}$ treatment with Gal1 (50 ng/ml), media was removed from each well, followed by addition of the dye binding solution (1X HBSS buffer) containing CyQUANT NF dye reagent (Fisher Scientific, Loughborough, UK). After 2-h incubation, the plates were read at Ex/Em: 485/530 using a FLUOstar BMG plate-reader (BMG Labtech Ltd, Bucks, UK) and cell proliferation was assessed based on the fluorescence intensity against the background containing HBSS buffer.

\section{HOMEC migration}

Assessment of cellular migration was carried out using a Cultrex Cell 96 transwell migration assay (Bio-Techne Ltd., Abingdon, UK) as previously described (5). Briefly, cells were incubated in growth factor-deprived media supplemented with $0.5 \%$ FCS overnight. Next, cells were seeded at a density of $5 \times 10^{4}$ in the upper assay chamber and treated \pm Gal1 $(50 \mathrm{ng} / \mathrm{ml})$ and in the presence or absence of ERK1/2 and/or AKT inhibitors at their given concentrations (Table 1). Negative controls received carrier alone. After $6 \mathrm{~h}$ incubation at $37^{\circ} \mathrm{C}$, the bottom chambers were washed, followed by addition of cell dissociation solution/calcein AM for a further hour to label and detach migrated cells. Fluorescence in the bottom wells was read at Ex/Em: 485/520 nm.

\section{Human galectin-1 ELISA (Bio-Techne Ltd., Abingdon, UK)}

HOMECs were treated \pm CathL \pm sulfasalazine $\left(\mathrm{NF}_{\kappa} \mathrm{B}\right.$ inhibitor, $100 \mu \mathrm{mol} / \mathrm{l}$ ) for $4 \mathrm{~min}, 30 \mathrm{~min}, 2,4,8$ and $24 \mathrm{~h}$. Supernatants were collected, centrifuged at $200 \mathrm{~g}$ for $10 \mathrm{~min}$, and transferred into fresh microfuge tubes which were either stored at $-20{ }^{\circ} \mathrm{C}$ for future experiments or immediately used to detect levels of Gal1. The assay was carried out according to the manufacturer's instructions.

\section{qRT-PCR}

Total RNA from cultured HOMECs treated with CathL \pm sulfasalazine was isolated with TRI reagent as per the manufacturer's protocol (Sigma-Aldrich, Gillingham, UK). Extracted RNA was DNase-treated using a DNAfree kit (Ambion, Northumberland, UK). Complementary DNA (cDNA) was synthesised from $1 \mu \mathrm{g}$ of RNA per sample using the qScript supermix kit (Quanta Biosciences, USA). Real-time RT-PCR was conducted using the TaqMan PrecisionPLUS master mix (Primer Design, Southampton, UK) with cycling conditions as follows: 1 
cycle of $60{ }^{\circ} \mathrm{C}$ for $2 \mathrm{~min}$ and $95^{\circ} \mathrm{C}$ for $2 \mathrm{~min}$; 40 cycles of $95^{\circ} \mathrm{C}$ for $10 \mathrm{~s}, 60^{\circ} \mathrm{C}$ for $30 \mathrm{~s}$; and 1 cooling cycle of $37^{\circ} \mathrm{C}$ for 30 s, on a LightCycler 96 Real-time Detection System (Roche, Welwyn Garden City, UK). The specificity of the PCR product was confirmed by melting-curve analysis. The Gal1, and two house-keeping genes GAPDH and $\beta$-2-microtubulin primers were purchased from Thermo Fisher Scientific (Northumberland, UK). For each gene, crossing point $(\mathrm{Cp})$ values were determined from the linear region of the amplification plot and normalized by subtraction of the geometric mean of the $C p$ values for two housekeeping genes. Relative expression was subsequently calculated using the $2-\Delta \mathrm{Cp}$ approach, and the data were presented as fold change in LGALS1 gene expression (normalised to control).

\section{Patients}

Female patients signed an informed consent form for the study which was reviewed by the Institutional Review Board (Gomel State Medical University), Gomel, Belarus. Sixty formalin-fixed and paraffin embedded archival tissue specimens including 20 cases of each of the following: normal omenta (from organ transplant donors), omenta of serous ovarian carcinoma patients without omental metastasis (SC wo MTS) and, with metastasis (SC w MTS), were used for immunohistochemistry (IHC). This was assessed by histopathologists D.A.Z. and S.L.A using the following criteria: a presence of papillary and solid pattern of tumour moderate to marked nuclear atypia, nuclear pleomorphism, stromal invasion, more than 12 mitotic figures in 10 high power fields [25]. Clinical information was obtained from the patients' medical records. None of the patients within the control group had tumours or infections, or previous peritoneal surgical operations and traumas. The group of HGSC (SC wo MTS) patients were selected based on the following criteria: absence of secondary tumours, absence of previous chemotherapy treatment (intraoperative diagnostic of cancer), absence of previous peritoneal surgical operations and traumas, stages II-III (FIGO, 2009). The clinicpathologic features of the ovarian serous carcinoma cases used in the present study are shown in Table 2. In stage II, cancer cells are found in other organs within the pelvis but not the omentum, and thus omenta from stage II patients were not included in the study [26].

\section{Immunohistochemistry}

Formalin-fixed, paraffin-embedded specimens were cut into $5 \mu \mathrm{m}$-thick sections and affixed to Thermo Super Frost slides (Thermo Fisher Scientific, Schwerte, Germany) and air-dried. This was followed by dewaxing in xylene and rehydration with descending graded alcohols to water. Tissue sections were immunostained
Table 2 Clinic-pathologic characteristics of patients

\begin{tabular}{lll}
\hline Characteristics & $\begin{array}{l}\text { SC wo MTS group } \\
(\mathbf{n = 2 0})\end{array}$ & $\begin{array}{l}\text { SC w MTS group } \\
(\mathbf{n}=\mathbf{2 0})\end{array}$ \\
\hline $\begin{array}{l}\text { Age } \\
\text { FIGO stage }\end{array}$ & $49.1(41.3-57.2)$ & $48.5(40.1-56.4)$ \\
IIA & 11 & - \\
IIB & 9 & - \\
IIIA & - & 10 \\
IIIB & - & 10 \\
Primary tumour grade & & 8 \\
Low grade & 9 & 12 \\
High grade & 11 & 12 \\
Ascites & & 8 \\
Absent & 19 & \\
Present $(>100 \mathrm{ml})$ & 1 & \\
\hline
\end{tabular}

using BenchMark XT (Roche-Ventana Medical Systems, Inc, Tucson, Arizona, USA). Heat-induced epitope retrieval was performed with Ventana Cell Conditioning 1 (Tris ethylenediaminetetraacetic acid buffer, $\mathrm{pH}$ 8.0) for $30 \mathrm{~min}$ on the instrument. The endogenous peroxidase activity was blocked with $3 \%$ hydrogen peroxide for $4 \mathrm{~min}$. Slides were incubated with or without (negative control) primary antibodies (anti-Gal1, Abcam, Cambridge, UK; anti-CD34, Roche-Ventana, Arizona, USA) for $30 \mathrm{~min}$ at room temperature. The subsequent reactions were performed using components from UltraView DAB Detection Kit (Roche-Ventana, Arizona, USA), including incubation with universal (rabbit and mouse) secondary antibody (or $8 \mathrm{~min}$, a biotin-free, horseradishperoxidase enzyme-labeled polymer, and a signal visualization by $3,3^{\prime}$-diaminobenzidine hydrochloride. Sections were then counterstained with hematoxylin [27].

\section{Analysis of immunohistochemistry staining}

Morphometrical analysis was carried out using a Nikon Eclipse 50i (Nikon, Japan) and NIS-Elements software (Nikon, Japan). To evaluate Gal1 expression, each case was rated according to a score that added a scale of intensity of staining to the area of staining [19]. At least five high-power fields (HPFs) were chosen randomly, and endothelium of every microvessel was counted separately. The intensity of Gal1 staining was graded on the following scale: 0 , no staining; $1+$, mild staining; $2+$, moderate staining; $3+$, intense staining.

To determine microvessel density as specified by Weidner, any CD34 brown staining endothelial cell or cell cluster that was clearly separated from adjacent microvessels, tumour cells and other connective tissue was considered a single countable microvessel [28]. Vessel lumens, although usually present, were not necessary for 
a structure to be defined as a microvessel, and red blood cells were not used to define a vessel lumen. Microvessels were determined blindly as the investigator was blinded to this variable. After selecting five microscopic fields of highest neovascularisation or hot spots, under low magnification, individual microvessels were counted manually and its area was counted using function "area" [29].

\section{Statistical analysis}

Data are expressed as mean \pm standard deviation (SD) and analysed using Mann-Whitney $U$ test and one-way ANOVA test. A Spearman (r) test with Chadock scale were used for correlation analysis. The univariate and multivariate hazard ratios (HR) were estimated using Cox regression analysis. A $p$ value of less than 0.05 was considered statistically significant. For all data, $n$ represents the number of patients, wells or dishes tested under each condition and also the results from at least two primary cell populations. Statistical analysis was performed using SPSS Statistical Software Package (SPSS Inc, Chicago, USA) and GraphPad Prism (GraphPad Software, California).

\section{Results}

\section{CathL induces secretion of Gal1}

Our recent data suggests a mitogen ligand-like activity for CathL in HOMECs [11], and our unpublished, preliminary data indicates that CathL induces a differential expression of galectin1 (Gal1) mRNA (LGALS1) in these cells. Therefore, we hypothesised that CathL induces production and secretion of Gal1. Figure 1a confirms that extracellular levels of Gal1 were, indeed, significantly increased in the presence of CathL between 30 min (twofold) and $8 \mathrm{~h}$ (5.2-fold) but returned to control levels after 24 h treatment (Fig. 1a). The latter may reflect Gall degradation since the protein has a reported serum half-life of $1.07 \mathrm{~h} \mathrm{[30]} \mathrm{or} \mathrm{re-uptake} \mathrm{by} \mathrm{the} \mathrm{cells} \mathrm{[31].} \mathrm{Interestingly,}$ Gal1 release was not induced in HOMECs treated with the other HGSC-secreted factors insulin-like growth factor binding protein 7 and CathD (data not shown), suggesting that Gal1 secretion may be CathL-specific.

\section{CathL induces upregulation of Gal1 mRNA expression}

As stated above CathL rapidly (within $30 \mathrm{~min}$ ) induced raised levels of extracellular Gal1, possibly reflecting secretion from intracellular stores or cleavage of cell surface Gal1 [32]. However, even greater levels of extracellular Gal1 were transiently detected after 4-8 h raising the possibility of additional transcriptional regulation (Fig. 1a). This was supported by the observation that $6 \mathrm{~h}$ CathL treatment also induced a transient, 1.5-fold, increase in LGALS1 mRNA level $(\mathrm{p}<0.05)$ vs control (Fig. 1b) following a time course that coincided with the increase in extracellular Gal1 levels. These data suggested that CathL induced a transient increase in LGALS1 mRNA expression, Gal1 protein production and then secretion in HOMECs, and thus the upstream signalling pathway was investigated further.

\section{CathL induces LGALS1 expression via activation of NFKB}

$\mathrm{NFKB}$ is a transcription factor involved in the production of many physiological and pathological proteins including Gal1 [22, 33] and thus, we examined the potential involvement of NFKB in CathL-induced Gal1 secretion in HOMECs. CathL-induced secretion of Gal1 was significantly reduced to control levels by sulfasalazine (inhibitor of $\mathrm{NFKB}_{\mathrm{K}}$ ) after $8 \mathrm{~h}$ treatment (0.9-fold vs 4.8 -fold (CathL-treatment), both normalised to control; Fig. 1c). These data not only suggest that CathL induces NFkB activation (confirmed in Fig. 1d), but that CathL induces transcriptional regulation of Gal1 via $\mathrm{NFKB}$ activation. This was further supported by the qRT-PCR data demonstrating that sulfasalazine significantly reduced LGALS1 mRNA expression in CathL-treated cells after $6 \mathrm{~h}$ treatment (Fig. 1e). Interestingly, sulfasalazine alone increased LGALS1 mRNA levels in HOMECs (Fig. 1e). This could

\footnotetext{
(See figure on next page.)

Fig. 1 CathL-induced Gal1 secretion in HOMECs is transcriptionally regulated via activation of NFKB. a Cells were seeded and starved overnight in media supplemented with 2\% FCS. Cells were then treated \pm CathL (50 ng/ml) and supernatants were collected after 4 min, 30 min, $2,4,8$ and $24 \mathrm{~h}$ treatment. A commercially available ELISA kit was used to assess the levels of extracellular Gal1 using a SpectraMax plate reader. Results are mean \pm SD and represented as fold change in secreted Gal1 vs control. $n=4-6 . \mathbf{b}$ Cells were treated \pm CathL (50 ng/ml) and lysed after 6 or $24 \mathrm{~h}$ treatment. Real-time PCR was performed on extracted RNA using a Roche LightCycler 96 and the data were normalised to GAPDH and $\beta 2$ M. c Gal1 secretion was assessed with or without CathL \pm sulfasalazine (100 $\mu \mathrm{mol} / \mathrm{l})$ for $8 \mathrm{~h}$ and the supernates were analysed as above. $\mathbf{d}$ CathL induces activation of NFKB (p65) in HOMECs. Cells were incubated with sulfasalazine (sulf, $100 \mu \mathrm{mol} / \mathrm{l}$ ) or media alone for $24 \mathrm{~h}$ and then treated \pm CathL $(50 \mathrm{ng} / \mathrm{ml})$ or positive control TNF-a $(160 \mathrm{pg} / \mathrm{ml})$ and in the absence or presence of sulfasalazine (100 $\mu$ mol/l). After $4 \mathrm{~h}$ treatment, the phosphorylated level of NFkB was assessed using a commercially available cell-based ELISA kit. Results are mean \pm SD and are represented as fold change in phosho-NFkB relative to total NFkB (compared to control). e CathL induces an upregulation of LGALS1 gene expression via NFkB activation. After overnight incubation and pre-incubation, cells were treated with CathL \pm sulfasalazine for $6 \mathrm{~h}$ and analysed for gene expression as above. Results are mean \pm SD and are represented as fold change in LGALS1 gene expression (relative to control), $n=4 .{ }^{*} p<0.05,{ }^{* *} p<0.01$ vs control. \#p<0.05, \#\#p <0.01 vs CathL. n.s. denotes not significant
} 
a

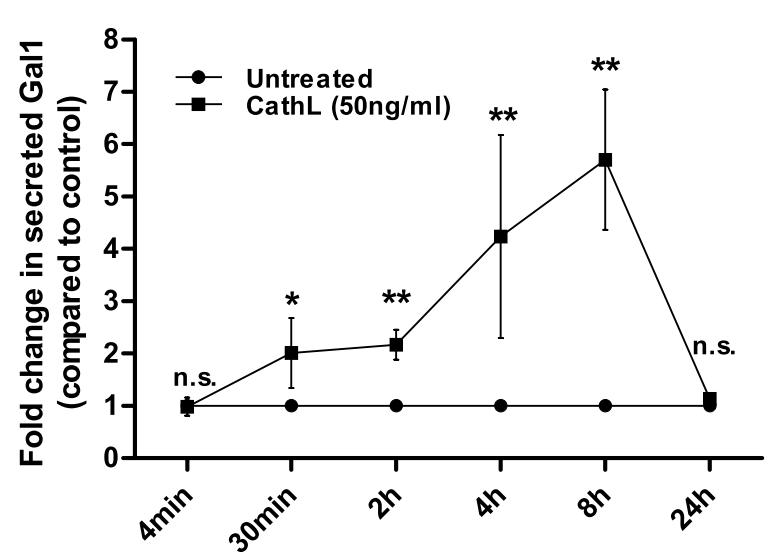

b

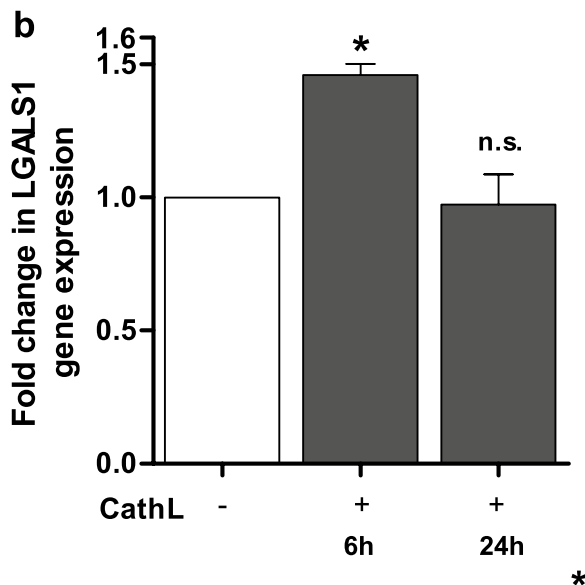

c

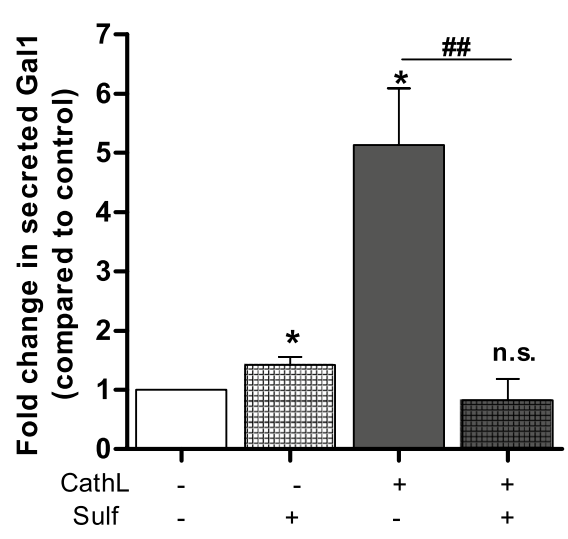

e

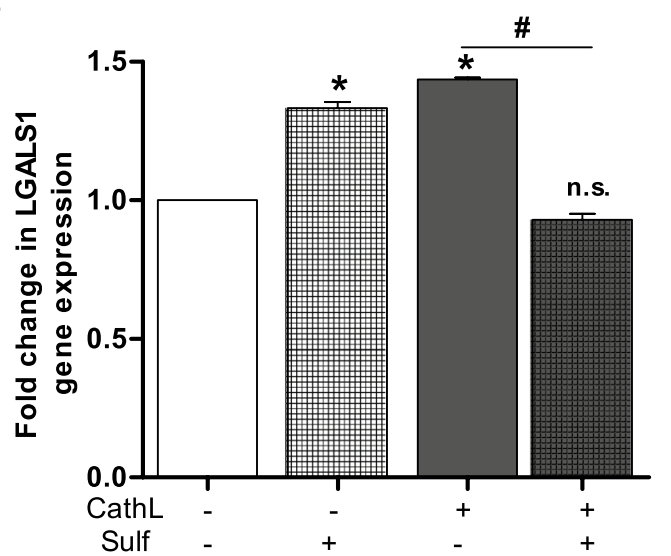

d

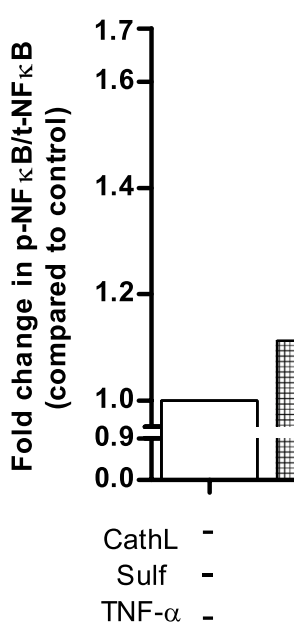

* 
HOMEC proliferation was initially investigated over a range of Gal1 concentrations based on the concentrations of Gal1 (20-80 $\mathrm{ng} / \mathrm{ml})$ released into the supernatant of CathL-treated ECs over $8 \mathrm{~h}$ (data not shown), and the median range concentration $(30-163 \mathrm{ng} / \mathrm{ml})$ of Gal1 in the sera of HGSC stage I patients [19]. Since, we are investigating induction of angiogenesis in ECs from noncancerous/non-metastatic omenta, we selected concentrations that fell within the lower spectrum of the in vivo range. Gal1 significantly induced cell proliferation at all concentrations tested vs control (100\%, WST- 1 assay) (Fig. 2a, Table 3). Additional studies with BrdU and CyQUANT proliferation assay kits confirmed that $50 \mathrm{ng} /$ $\mathrm{ml}$ Gal1 significantly enhanced proliferation vs control (100\%) (Fig. 2b, c). On the basis of these results and the levels of secreted Gal1, $50 \mathrm{ng} / \mathrm{ml}$ of Gall was selected for further experiments.

The pro-proliferative role of Gal1 in HOMECs suggested possible activation of intracellular signalling pathways downstream of Gal1-cell surface interaction. We hypothesised activation of the ERK $1 / 2$ and PI3K/AKT pathways based on our previous publications $[8,11]$ in these cells. Gal1 treatment rapidly $(<4 \mathrm{~min})$ increased phosphorylation of ERK1/2 (1.8-fold vs control, Fig. 2d), with phosphorylated levels decreasing to control levels after $10 \mathrm{~min}$ (Fig. 2e); suggesting a transient activation. Similar results were seen for AKT, with phosphorylation transiently increased (1.7-fold vs control; Fig. 2f, g) within $4 \mathrm{~min}$ of Gal1 treatment. The ELISA data were verified using specific inhibitors of ERK1/2 (U0126 and PD98059) and PI3K/AKT (LY294002 and MK2206) using previously determined concentrations (Additional file 1: Figure S1) $[8,11]$.

Since Gal1 induced activation of the ERK1/2 and PI3K/ AKT pathways, we tested the effect of inhibition of these pathways on Gal1-induced HOMEC proliferation. Both ERK1/2 inhibitors, U0126 and PD98059, significantly reduced Gal1-induced cell proliferation $(\mathrm{p}<0.001$, Fig. 3a). Gal1-induced HOMEC proliferation was also significantly reduced by the PI3K inhibitor, LY294002 $(\mathrm{p}<0.001)$ but the selective AKT inhibitor MK2206 had no effect (Fig. 3b, discussed later).

\section{Gal1 induces migration in HOMECs}

A second key element of angiogenesis is EC migration and thus, the pro-migratory effect of Gal1 and the pathways involved were examined using inhibitors of ERK1/2 and AKT as above. Interestingly, although Gal1 significantly induced HOMEC migration $(163.4 \pm 47.2 \%$ vs control, 100\%) none of the inhibitors tested significantly reduced this (Fig. 4a, b). These data combined with the ELISA data (Additional file 1: Figure S1) suggest that Gal1-induced HOMEC migration may not solely depend on the activation of the ERK $1 / 2$ and AKT pathways, and that other kinases may be involved.

\section{High positive correlation between in vivo Gal1 expression and number and area of vessels - a potential proangiogenic role for Gal1 in HOMECs}

The in vitro data presented above suggest that HOMECsecreted Gal1 may play a role in pro-angiogenic changes in the omental microvasculature. To examine this further in human disease we immunohistochemically investigated expression of Gal1 protein levels in the microvascular endothelium in patient samples; specifically, in normal omenta (control), omenta with HGSC metastasis (SC w MTS) and omenta with no metastatic tumour (SC wo MTS).

Interestingly, although the microvessels stained positively for Gal1 in all groups (Fig. 5a), the intensity of Gal1 expression (designated 1-3, see supplementary methods and [19]) differed between the groups. In the control, SC wo MTS and SC w MTS groups, Gal1 mean scores were $1.32 \pm 0.29,1.42 \pm 0.37$ and $2.39 \pm 0.28$ respectively with statistically significant differences $(\mathrm{p}<0.001)$ between the control and the SC w MTS group, and the SC wo MTS and SC w MTS groups (Fig. 5b).

\footnotetext{
(See figure on next page.)

Fig. 2 Gal1 induces HOMEC proliferation and activation of ERK1/2 and AKT. Cells were seeded in 2\% gelatin pre-coated 96 well plates at a density of 10,000 cells/well in starvation media containing $2 \%$ FCS. a-c After overnight incubation, cells were treated \pm Gal 1 at various concentrations and incubated for $48 \mathrm{~h}$. a WST-1 assay was used to assess cellular proliferation based on absorbance using a PHERAstar BMG plate-reader at $450 \mathrm{~nm}(\mathrm{n}=20)$. $\mathbf{b}$ Cell proliferation was examined at $50 \mathrm{ng} / \mathrm{ml}$ of Gal1 (CyQUANT). A commercially available CyQUANT reagent was used to assess cell proliferation after $48 \mathrm{~h}$ treatment based on fluorescence intensity using a FLUOstar BMG plate-reader at Ex/Em: 485/530 nm ( $n=20)$. c A commercially available BrdU reagent was added to the wells for the last $24 \mathrm{~h}$ incubation and cellular proliferation was assessed (according to the manufacturer's instructions) at $48 \mathrm{~h}$ based on absorbance using a SpectraMax plate-reader at 450/550 $\mathrm{nm}(\mathrm{n}=15)$. Results are mean \pm SD and shown as percentage of the control, ${ }^{* *} p<0.01$ and ${ }^{* * *} p<0.001$ vs control (100\%). After overnight incubation, cells were treated $\pm 50 \mathrm{ng} / \mathrm{ml}$ of Gal1 or $20 \mathrm{ng} / \mathrm{ml}$ of VEGF and incubated for 4 or $10 \mathrm{~min}$. ERK1/2 (d, e) and AKT (f, $\mathbf{g})$ phosphorylation was examined after $4 \mathrm{~min}(\mathbf{d}, \mathbf{f})$ and $10 \mathrm{~min}(\mathbf{e}, \mathbf{g})$ treatments. Commercially available cell-based ELISAs were used for the determination of ERK1/2 and AKT (S473) phosphorylation level. The ELISA experiments were carried out in quadruplets on two cell batches. The data show fold change in phosho-ERK1/2/AKT relative to total ERK1/2/AKT (compared to control). Results are mean $\pm S D, n . S .,{ }^{*} p<0.05,{ }^{* *} p<0.01$ vs control (dotted lines); $n=4-6$. n.s. denotes not significant vs control
} 


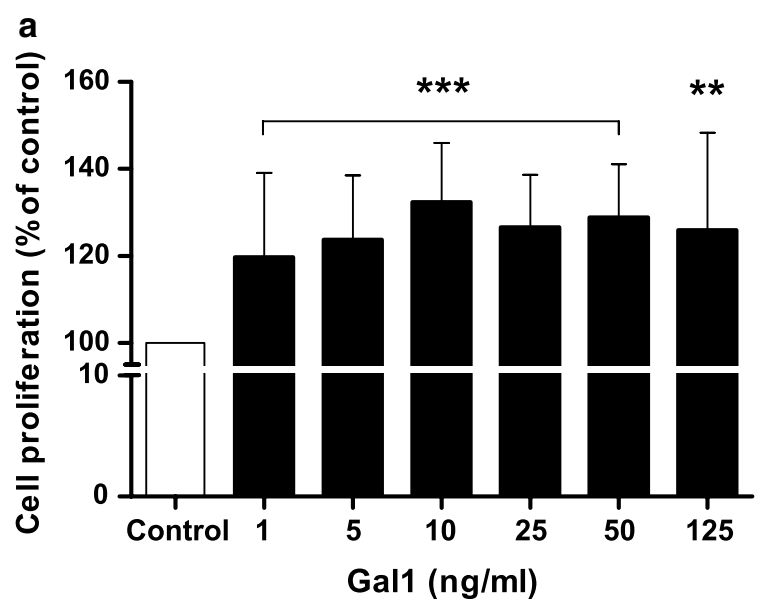

C
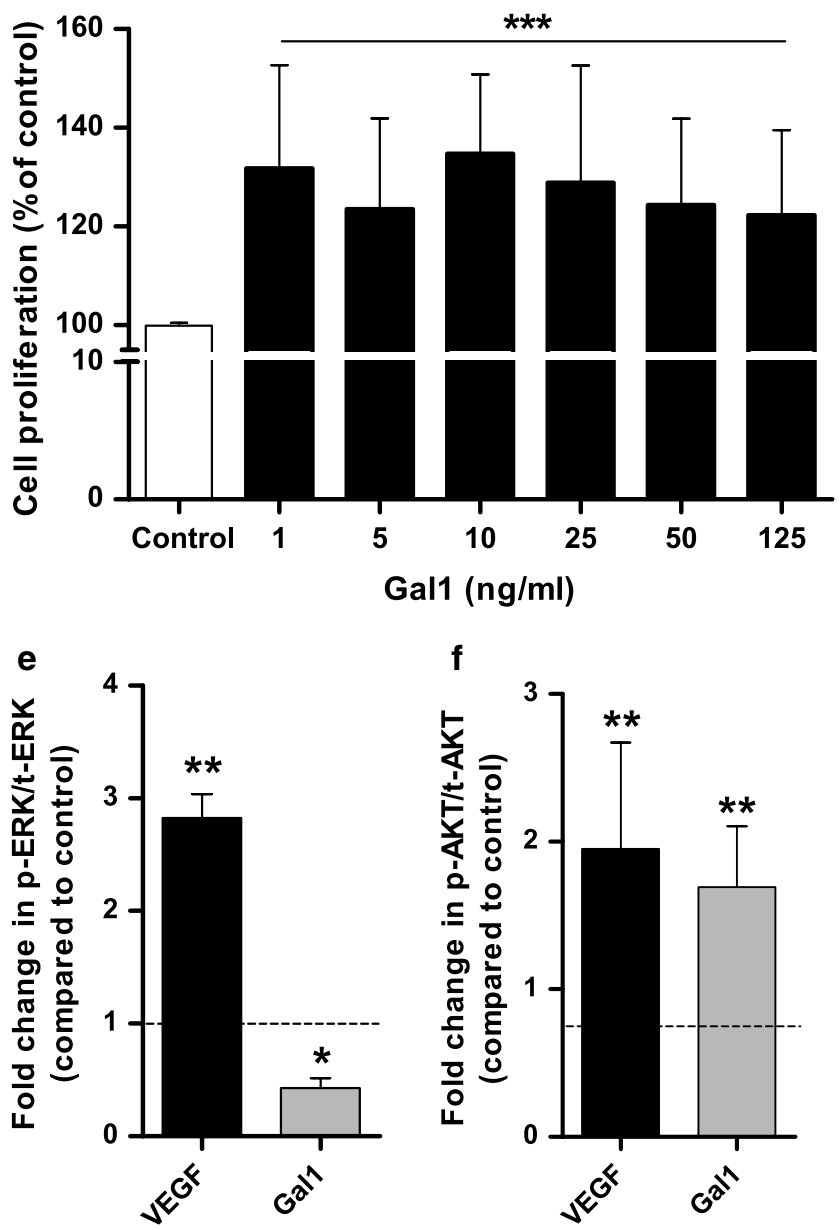

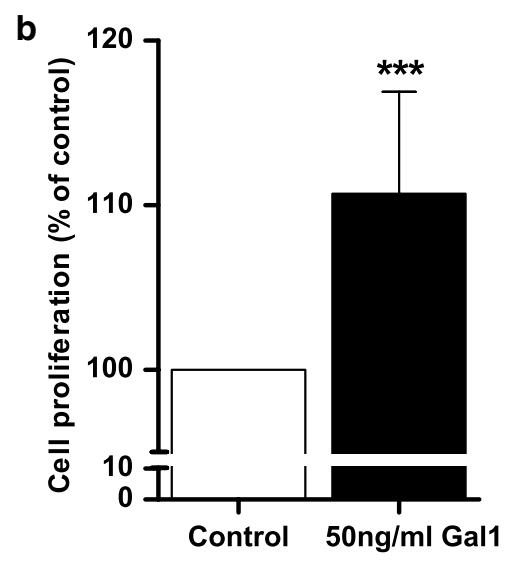

d
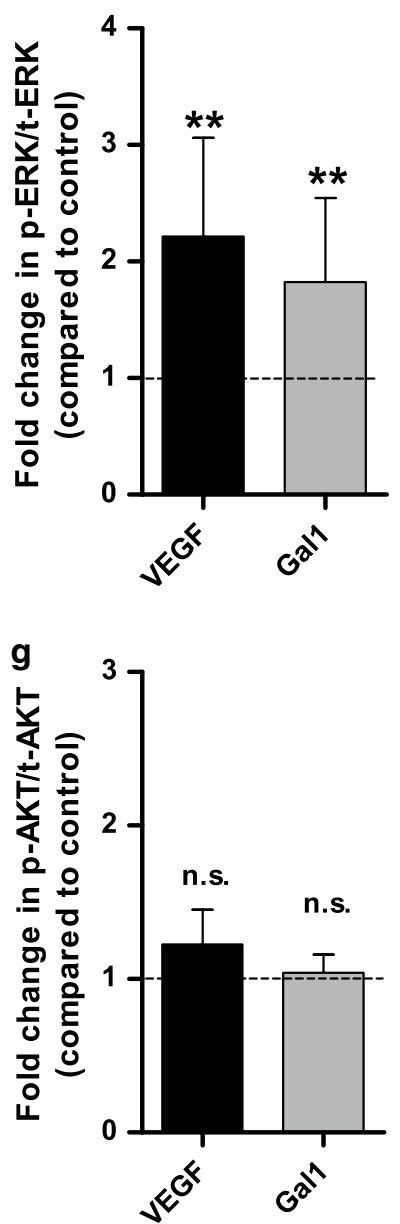

Additionally, we investigated microvessel density (as assessed by CD34 staining of the endothelium), a measure of vascularisation i.e. angiogenesis, in each group (Fig. 5a). There was a significant increase $(\mathrm{p}<0.001)$ in vessel density in the SC $\mathrm{w}$ MTS group compared with both the control and SC wo MTS groups (Fig. 5c), confirming a significant increase in microvessel density in the omenta invaded by tumour. No non-specific staining was observed in our negative controls of each group (Additional file 2: Figure S2). 
Table 3 Summary of the pro-proliferative effect of Gal1 on HOMECs at various concentrations (WST1 assay) (shown in Fig. 3a)

\begin{tabular}{llllllll}
\hline & Ctrl\% & \multicolumn{6}{l}{ HOMEC proliferation (as \% of control) at each Gal1 concentration (ng/ml) } \\
\cline { 3 - 8 } & $\mathbf{1}$ & $\mathbf{5}$ & $\mathbf{1 0}$ & $\mathbf{2 5}$ & $\mathbf{5 0}$ & $\mathbf{1 2 5}$ \\
\hline $48 \mathrm{~h}$ & 100 & $119.7 \pm 18.7$ & $123.8 \pm 14.8$ & $132.3 \pm 13.6$ & $113.2 \pm 13.7$ & $128.9 \pm 12.2$ & $125.9 \pm 22.4$ \\
\hline
\end{tabular}

HOMECs were \pm increasing concentrations of Gal 1 for $48 \mathrm{~h}$. Results are mean \pm SD and shown as percentage of control (100\%). $\mathrm{n}=11-20$

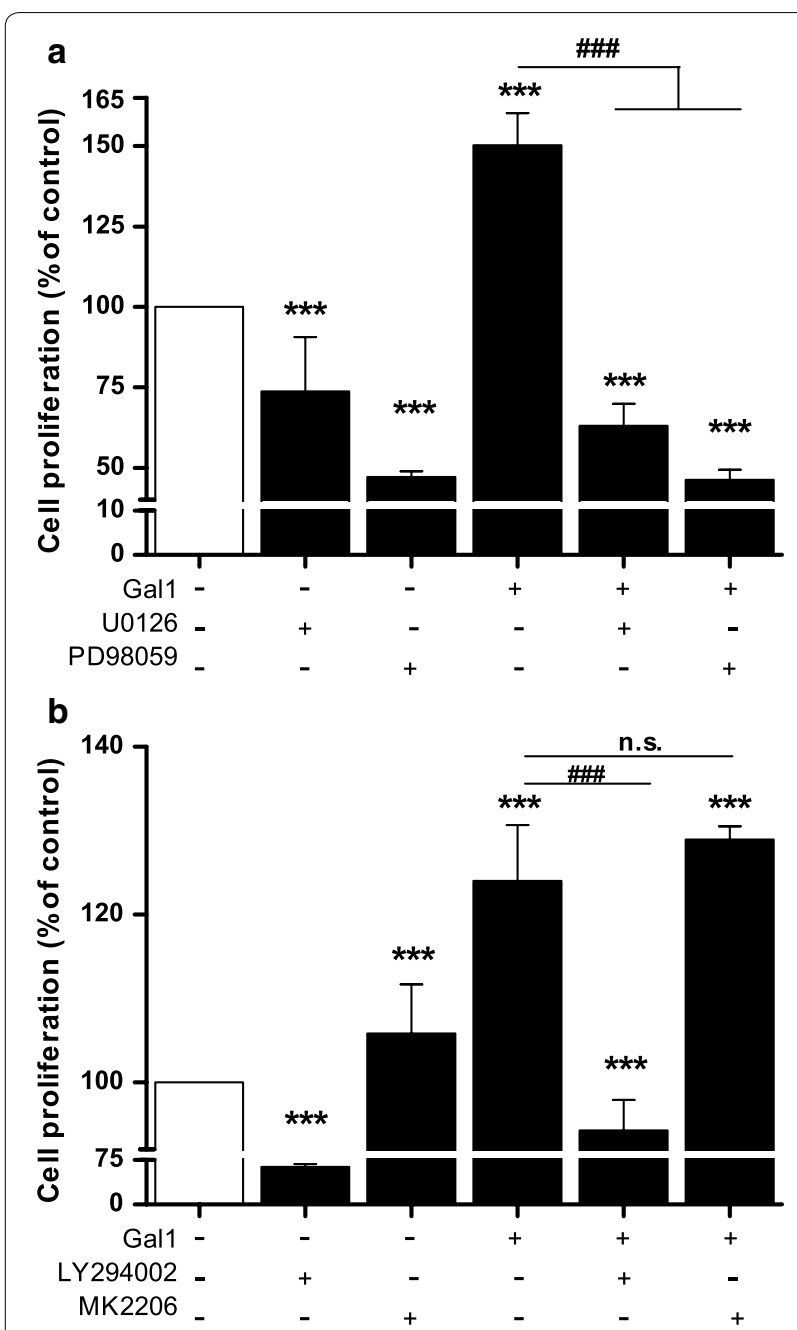

Fig. 3 Gal1 induced HOMEC proliferation via the ERK1/2 and PI3K pathways, but not AKT pathway. Cells were seeded in $2 \%$ gelatin pre-coated 96 well plates at a density of 10,000 cells/well in starvation media containing 2\% FCS. After overnight incubation, cells were treated \pm Gal $1(50 \mathrm{ng} / \mathrm{ml})$ and in the absence or presence of $\mathbf{a} \cup 0126$

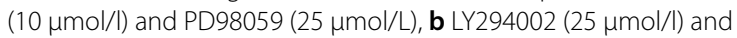
MK2206 (5 $\mu \mathrm{mol} / \mathrm{l})$ and incubated for $72 \mathrm{~h}$. Commercially available WST-1 assay was used to assess cellular proliferation. Results are mean \pm SD and shown as percentage of the control, ${ }^{* *} p<0.001$ vs control (100\%), \#\#\#p<0.001 vs Gal1 (normalised to control 100\%), $n=14-15$. n.s. denotes not significant
We also measured area of vessels (per $1 \mathrm{~mm}^{2}$ ) and found a significant increase in microvessel area $(\mathrm{p}<0.001)$ in the SC w MTS group compared with SC w/o MTS and control groups (Fig. 5d).

An association study revealed a positive correlation between Gal1 and number of vessels (Spearman coefficient, $\mathrm{r}=0.8702, \mathrm{p}<0.001)$, and area of vessels $(\mathrm{r}=0.7283, \mathrm{p}<0.001)$ (Fig. 5e), suggesting a potential proangiogenic role for Gal1 in the omentum of advanced ovarian carcinoma.

Univariate Cox regression analysis of prognosis factors showed a significant association between predictive patient survival and Gal1 expression $(\mathrm{p}<0.001)$ and number of vessels $(p=0.032)$, but not area of vessels (Fig. $5 f)$; possibly due to atypical vessels - a phenomenon seen in tumour invasion. Interestingly, in multivariate Cox regression analysis only Gal1 expression $(\mathrm{p}<0.001)$ (but not area or number of vessels) demonstrated significant prognostic value in ovarian cancer patients (Fig. 5g), suggesting that Gal1 expression could be a prognostic factor in advanced ovarian cancer with a role in angiogenesis. These data all strongly support a proangiogenic role for Gall in HGSC metastasis to the omentum.

\section{Discussion}

Treatment of HGSC remains challenging due to late diagnosis and limited effective therapeutic strategies for advanced metastatic disease. This is compounded by the lack of understanding of the molecular mechanisms involved in the establishment and growth of the metastases; in particular the angiogenic activators that transform the local, host microvascular endothelium into a pro-angiogenic state. Targeting this process is an attractive therapeutic strategy. Indeed, previous therapies have concentrated on disruption of the VEGF pro-angiogenic pathway e.g. bevacizumab. However, these strategies have had disappointing results [3-7], highlighting the need to identify new potential targets for HGSC e.g. alternative proangiogenic factors.

One of the major sites of HGSC metastasis is the omentum and, we have previously reported that omental 


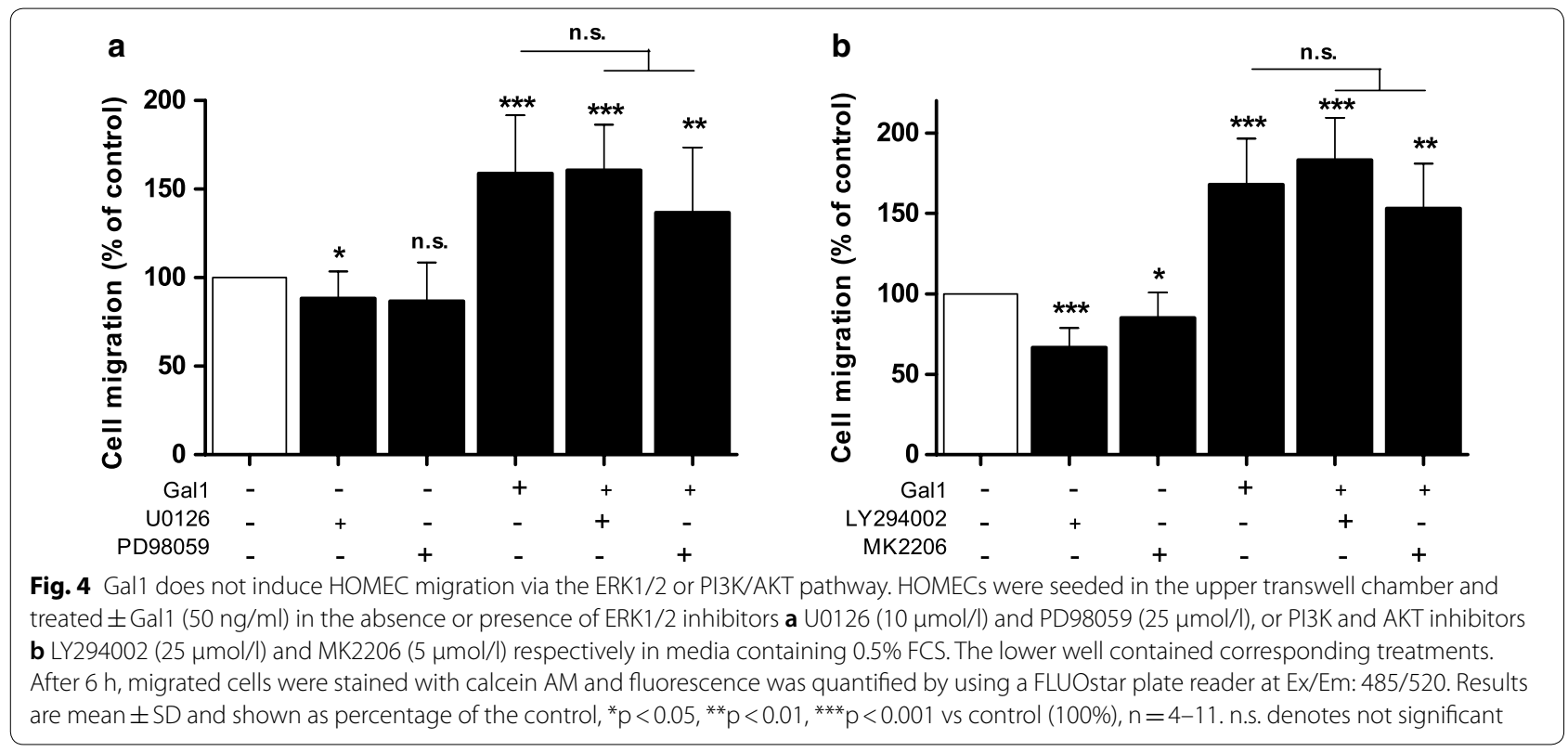

angiogenesis during metastasis of HGSC may occur independently of the VEGF/VEGFR axis. Specifically, we showed that CathD and CathL, two HGSC-secreted enzymes, significantly induced proangiogenic endothelial responses in omental ECs (HOMECs) $[8,11]$. Here we show that CathL may induce secretion of Gall from HOMECs which acts as an autocrine pro-angiogenic factor in the local microvasculature, contributing to secondary tumour growth. One important aspect of these studies is the use of omental microvascular ECs. Since ECs display heterogeneity in their proteomics, morphology and functionality, it is essential to undertake studies using cells from a relevant vascular bed.

Gal1 has been shown to be involved in tumour progression, development and angiogenesis [21, 23]. However, a role for CathL-induced extracellular Gal1 in HGSC metastasis is a novel finding. The observation that CathL-induced increases in extracellular Gall levels were detected within 30 min suggests release from cytoplasmic stores [12, 32]. The enhanced CathL-induced cellular release of Gal1 at 4 and $8 \mathrm{~h}$ suggested an additional transcriptional regulation of LGALS1 which was confirmed by increased expression of LGALS1 with CathL treatment. Although CathL has not previously been reported to induce expression of LGALS1 or secretion of Gal1, transcriptional regulation of Gal1 has been reported in ECs, specifically, LDL-induced expression of Gal1 in human aortic ECs [34].

CathL significantly enhanced NFkB phosphorylation and the NFkB inhibitor, sulfasalazine, significantly reduced this phosphorylation as well as LGALS1 expression and Gal1 secretion, supporting a role for NFkB in CathL-induced Gal1 secretion. We reported previously that CathL acts non-proteolytically on these cells and induces proangiogenic changes. Thus, it is possible that CathL binds to an, as yet, unidentified receptor and induces this pathway.

Next, we showed that Gall acted as a potent proangiogenic factor in HOMECs, stimulating both proliferation and migration; an observation that agrees with other findings showing pro-proliferative effects of exogenous Gal1 in human ECs [23, 31]. We then investigated

\footnotetext{
(See figure on next page.)

Fig. 5 High positive correlation between Gal1 expression and number of vessels, and area of vessels. a Expression of Gal1 (upper panel) and CD34 (lower panel) in endothelium (moderate expression- red arrows) in control (left), SC wo MTS (serous carcinoma without omental metastasis, moderate expression; middle), and SC w MTS (serous carcinoma with omental metastasis, high expression; right). In SC w MTS, Gal 1 expression is shown in endothelium (red arrows), cancer cells (orange arrows) and stromal lymphocytes infiltration (yellow arrows). b Significantly higher Gal1 expression in SC w MTS group compared to control and SC wo MTS. Gal1 expression is represented as an intensity score, as described by Chen et al. [19]. c A significant increase in the number of vessels and $\mathbf{d}$ area of vessels were found in SC w MTS group compared to the other groups. The vessels were counted according to Weidner's method described in "Materials and method" section [28]. e Very high correlation between Gal1 and number, and area of vessels, indicating a potential proangiogenic role for Gal1. $\mathbf{f}$ Univariate and $\mathbf{g}$ multivariate hazard ratios of Cox analysis of predictive patients' survival. Photographs were taken using a Nikon Eclipse 50i. ${ }^{* *} \mathrm{p}<0.001$ vs control group, \#\#\#p $<0.001$ vs SC wo MTS. n.S. denotes not significant. Magnification: $\times 200$
} 


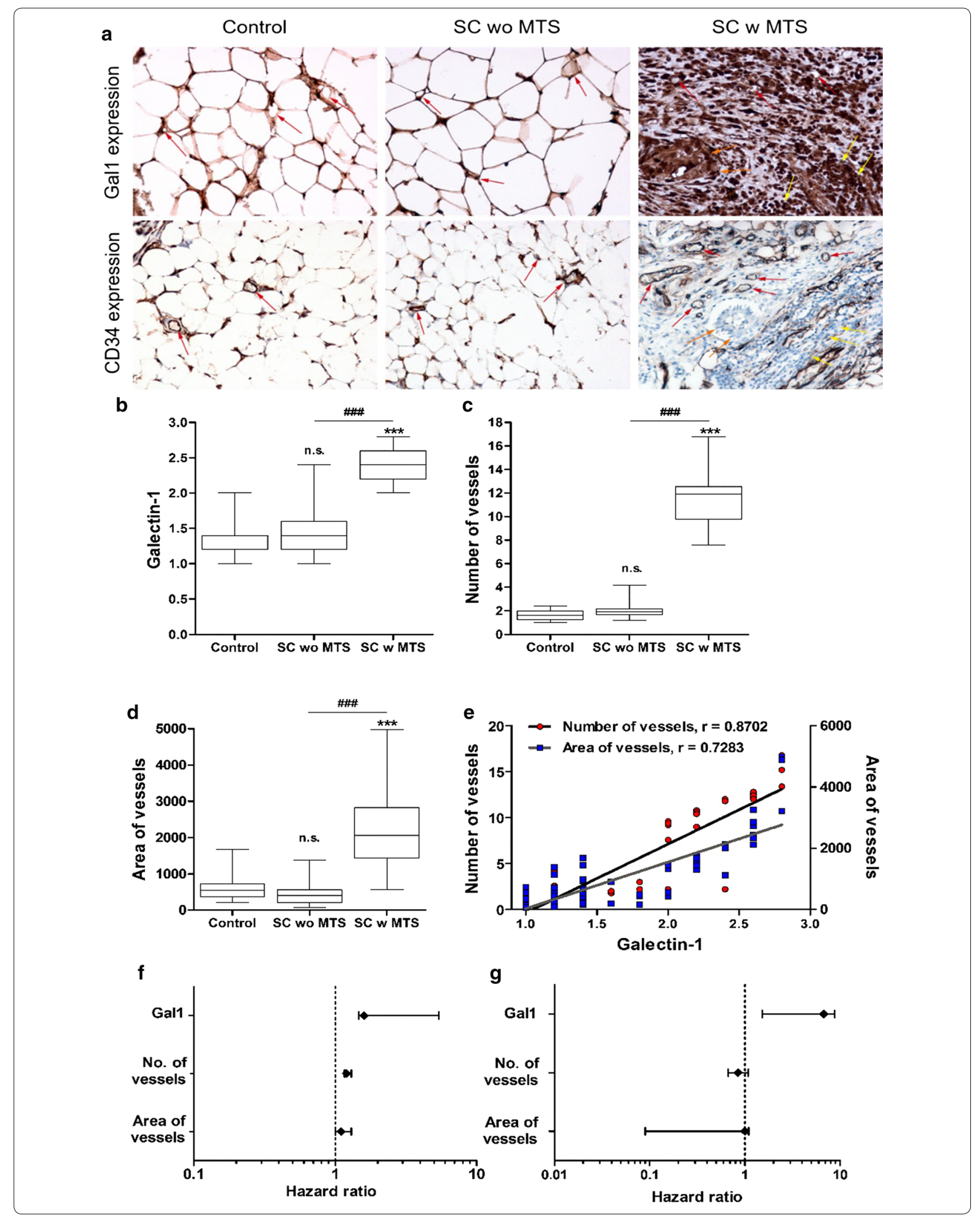


the potential signalling pathways activated downstream by Gal1; concentrating on two key signalling molecules ERK1/2 and AKT. Gal1 induced significant and transient phosphorylation of both, an effect which was abolished by inhibitors of MEK1/2 (upstream of ERK1/2, U0126 and PD98059) and PI3K/AKT (LY294002 and MK2206). Gal1-induced HOMEC proliferation was significantly reduced by the two MEK1/2 inhibitors and the PI3K inhibitor LY294002, but not the selective AKT inhibitor MK2206. It is possible that the latter result was observed due to cross-reactivity of LY294002 with the ERK1/2 pathway and thus inhibition of ERK1/2 phosphorylation [35]. Taken together, our data suggest that ERK1/2 pathway is involved in the HOMEC proliferation induced by exogenous Gal1 (Fig. 6).

Gal1 also had a potent pro-migratory effect on HOMECs. However, this was not impacted by the ERK1/2 or AKT inhibitors despite previous reports that these kinases are involved in cell migration $[8,11]$. In ECs Thijssen et al. [31] previously suggested involvement of activated ERK1/2 in Gal1-induced HUVEC migration, however Hsieh et al. [36], reported that activated JNK, a

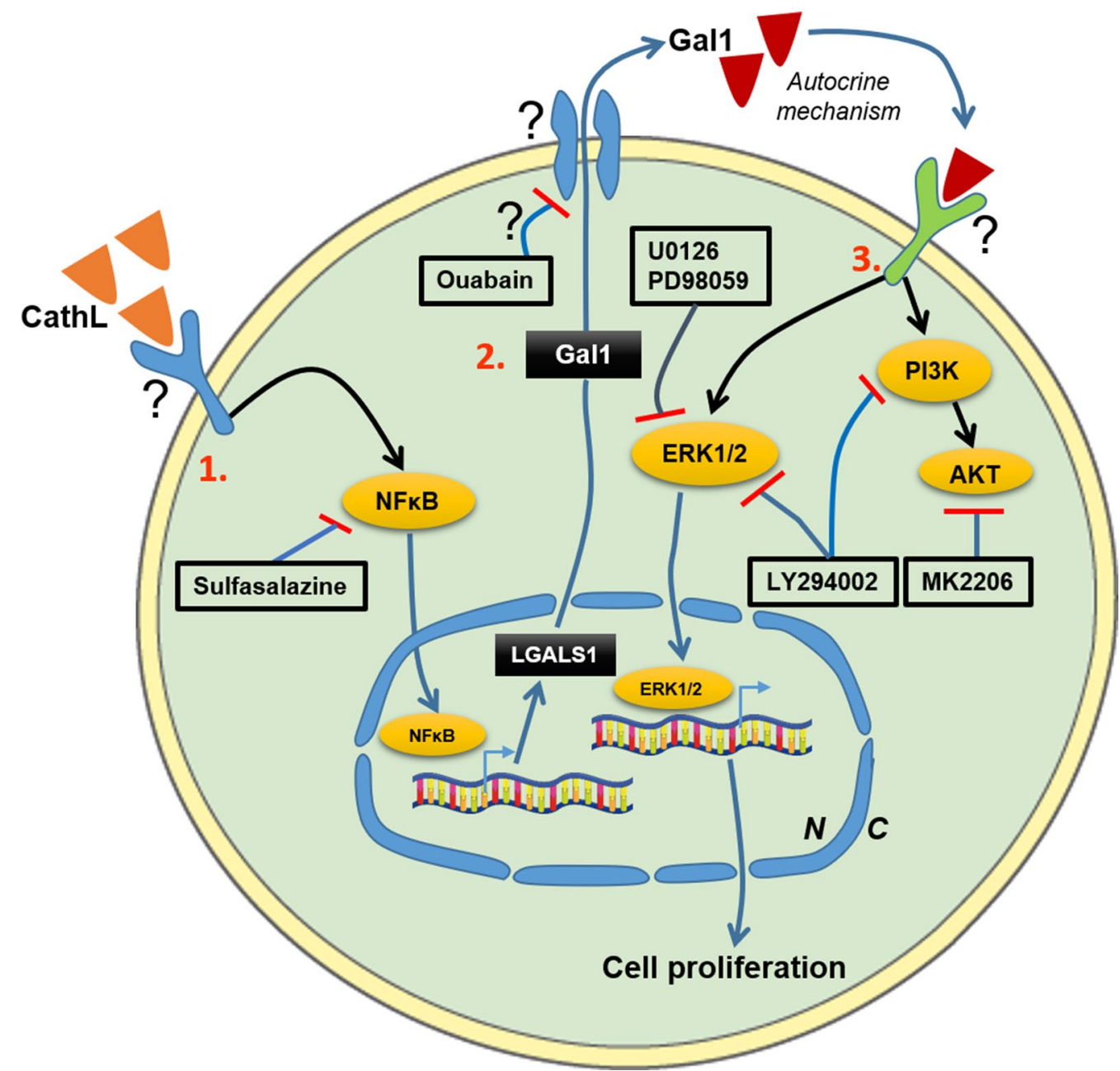

Fig. 6 A summary of the proposed CathL-induced Gal1 secretion pathway and its pro-proliferative function and potential therapeutic targets in HOMECS. 1. CathL non-proteolytically activates a cell surface receptor, possibly a receptor tyrosine kinase, which leads to an increase in activated levels of the transcription factor NFKB, resulting in increased expression of LGALS1 (Gal1 mRNA). Sulfasalazine, an inhibitor of NFKB, reduces LGALS1 expression and subsequent secreted level of Gal1. 2. LGALS1 is transcribed and translated into Gal1 which is secreted out of the cell, possibly utilising a $\mathrm{Na}^{+} / \mathrm{K}^{+}$ATP-pump, via a non-conventional secretory pathway. 3. Secreted Gal1 induces activation of MAP kinase ERK1/2 and PI3K/AKT via an autocrine mechanism and an unknown receptor(s), where the ERK1/2 pathway induces HOMEC proliferation. The MEK/ERK1/2 inhibitors U0126 and PD98059 significantly reduce this cellular function by inhibiting ERK1/2 phosphorylation. Both the PI3K inhibitor LY294002 and AKT inhibitor MK2206 inhibit phosphorylation of AKT at Ser473 (S473) in Gal1-treated HOMECs. However, only LY294002, but not MK2206, inhibits Gal1-induced HOMEC proliferation, suggesting a cross-reactivity of PI3K inhibitor LY294002 with the ERK1/2 pathway. Potential therapeutic targets are: Gal1 secretory mechanism, Gal1 binding to its receptor(s), and blocking receptor(s) for Gal1. N, nucleus; C, cytoplasm 
MAP kinase, is responsible for Gal1-induced migration in HUVECs, highlighting the potential involvement of other kinases in HOMEC migration.

We then carried out immunohistochemical analysis of patient samples to complement the in vitro studies. Although adipocytes are known to express Gal1 on their cell membrane [37], strikingly, there was a significant increase in Gall expression in the microvessel endothelium and surrounding stromal cells in the omenta with metastatic serous carcinoma compared with normal or non-metastatic serous carcinoma omenta, with a strong positive correlation between Gal1 staining intensity and both microvessel number and vessel area. A significant Gal1 expression and its role in angiogenesis and metastasis has also been reported in cancer-associated stroma in primary ovarian tumour, breast and pancreatic cancers [38-40]. Here, we show high-intensity Gal1 expression in cancer-associated stroma in secondary lesions for the first time in the metastasised omenta. These data, support the possibility that Gal1 is a potent proangiogenic factor in HGSC. Since we previously showed increased endothelial expression of CathL in the omenta of patients with metastasised serous carcinoma [41], the current observation of a similar increased Gal1 expression pattern in comparable patient samples could signify a physiological role for CathL induction of Gal1 expression in angiogenesis in this pro-metastatic disease.

We also demonstrated a significant association between increased Gal1 expression and a poor predictive survival for HGSC patients. Interestingly, Schulz and colleagues reported that overexpression of Gal1 was associated with increased migratory and invasive behaviour, and decreased sensitivity to cisplatin, in HGSC cells; possibly explaining the decreased survival of HGSC patients with increased Gal1 expression [42].

In summary, our data suggest that the HGSC-secreted factor CathL induces secretion of Gall from HOMECs which, via an autocrine mechanism, stimulates pro-angiogenic cellular responses i.e. proliferation and migration in the omental vasculature during metastasis of HGSC.

Our observation that Gal1 may play an important proangiogenic role is supported by previous reports indicating a role for Gal1 in tumour progression, partly by triggering angiogenesis [20]. For instance, in vitro, elevated Gal1 induced HUVECs to migrate and proliferate and in in vivo studies, Gal1 knockout mice had severely compromised tumour growth and vessel density [21]. In a variety of cancers including prostate, colon and thyroid [17], Gal1 is highly expressed in cancer-associated stroma, and this over-expression correlates with pathological factors including advanced disease stage, tumour invasion and increased rates of disease reoccurrence [19]. Over-expression of Gal1 has also been found to increase tumour cell invasion in the OVCAR-3 cell line [19]. Moreover, when the serum levels of Gal1 were compared in ovarian cancer patients alongside the most widely used biomarker CA125, it was found that around $70 \%$ of patients were correctly identified as positive by both proteins [19], suggesting that Gal1 could potentially be used as a biomarker for HGSC progression, as well as outcome.

\section{Conclusions}

Identification of new targets is a priority in advanced HGSC. Our data indicate a strong proangiogenic effect of Gal1 on omental ECs, with high positive correlation between Gal1 and microvessel density in vivo, highlighting that the Gall pathway could be a potential therapeutic target in HGSC metastasis to the omentum. Although anginex, a non-specific anti-angiogenic compound, has been shown to reduce angiogenesis by binding and possibly blocking cell membrane-bound Gal1 [21, 43], there is currently no FDA-approved therapy in place to block potent effects of secreted Gal1. However, there are a number of potential Gal1-related cellular targets [44] (Fig. 6), for instance, the secretory pathway of Gal1 and potential Gal1-binding receptors that could potentially be targeted in the quest to find an effective treatment for metastasised HGSC.

\section{Additional files}

Additional file 1: Figure S1. Inhibitors of ERK1/2 and AKT reduce ERK1/2 and AKT phosphorylation respectively in intact HOMECs. After overnight incubation in starvation media (containing $2 \% \mathrm{FCS}$ ), cells were preincubated with the ERK1/2 inhibitors a) U0126 (10 $\mathrm{mmol} / \mathrm{l})$ and PD98059 $(25 \mu \mathrm{mol} / \mathrm{l})$ or PI3K/AKT inhibitors b) LY294002 $(25 \mu \mathrm{mol} / \mathrm{l})$ and c) MK2206 ( $5 \mu \mathrm{mol} / \mathrm{l})$ for (a) 20-30 min or ( $\mathrm{b}+\mathrm{c}$ ) $2.5 \mathrm{~h}$, and then co-treated with or without $50 \mathrm{ng} / \mathrm{ml}$ of Gal1 or $20 \mathrm{ng} / \mathrm{ml}$ of VEGF for $4 \mathrm{~min}$. Commercially available cell-based ELISAs were used for determination of phosphorylation levels. The data show fold change in phospho-protein relative to total protein (compared to control). Results are mean $\pm S D,{ }^{*} p<0.05$, ${ }^{* *} p<0.01$ vs control (1-fold, dotted lines), \#p<0.05 vs VEGF/Gal 1 (normalised to control), $n=4$.

Additional file 2: Figure S2. Tissue sections were stained only with secondary antibody to both Gal1 and CD34 in control group, groups with serous carcinoma without metastasis (SC wo MTS) and with metastasis (SC w MTS). Photographs were taken using a Nikon Eclipse 50i. Magnification $\times 200$.

\section{Abbreviations}

AKT(S473): protein kinase B; CathD: cathepsin D; CathL: cathepsin L; CDNA: complimentary DNA; EC: endothelial cell; EOC: epithelial ovarian cancer; ERK1/2: extracellular signal-regulated kinases 1/2; FCS: foetal calf serum; Gal1: galectin-1; HOMECs: human omental microvascular endothelial cells; HPF: high-power field; HUVECs: human umbilical vein endothelial cells; JNK: c-Jun N-terminal kinases; LGALS1: galectin-1 mRNA; MAP: mitogen-activated protein kinase; MEK: mitogen-activated protein kinase kinase; NFkB: nuclear factor kappa-light-chain-enhancer of activated B cell; PBMCs: peripheral blood mononuclear cells; SC w MTS: serous carcinoma with metastasis; SC wo MTS: 
serous carcinoma without metastasis; TNF-a: tumour necrosis factor-alpha; VEGF: vascular endothelial growth factor.

\section{Acknowledgements}

We thank Boleslaw Winiarski for his intellectual input and help with HOMEC isolation and cell culture and Erica Hossain for her help with drawing the figures. This project was supported by the NIHR Exeter Clinical Research Facility.

\section{Authors' contributions}

MZIP and JLW designed the research. MZIP and DAZ performed the study, conducted the experiments and analysed the data. ARTM, LJS, SLA, DML and EAN performed parts of the experiments. MZIP, DAZ and JLW wrote the manuscript. MH, NJG and JLW reviewed the study and the manuscript. All authors read and approved the final manuscript.

\section{Funding}

This project was funded by FORCE Cancer Charity, Devon (Grant 50703, Charity registration no. 1140676).

\section{Availability of data and materials}

The research materials supporting this publication can be accessed by contacting Jacqueline Whatmore (J.L.Whatmore@exeter.ac.uk).

\section{Ethics approval and consent to participate}

Fresh omental samples were obtained with ethical approval and informed consent from patients undergoing surgery at the Royal Devon and Exeter NHS Foundation Trust (Exeter, UK). The immunohistochemical study was undertaken at the Gomel State Medical University, Belarus with informed consent from all patients, with ethical approval from the Institutional Review Board.

\section{Consent for publication}

All the authors have read and approved the manuscript.

\section{Competing interests}

The authors declare that they have no competing interests.

\section{Author details}

${ }^{1}$ Institute of Biomedical and Clinical Science, University of Exeter Medical School, Exeter, Devon EX1 2LU, UK. ${ }^{2}$ William Harvey Research Institute, Barts and the London School of Medicine and Dentistry, Queen Mary University of London, London EC1M 6BQ, UK. ${ }^{3}$ Department of Pathology, Gomel State Medical University, 246000 Gomel, Belarus. ${ }^{4}$ Department of Anatomical Pathology, Gomel Regional Clinical Oncological Dispensary, 246012 Gomel, Belarus. ${ }^{5}$ Gomel Regional Clinical Oncological Dispensary, 246012 Gomel, Belarus. ${ }^{6}$ Royal Devon and Exeter NHS Foundation Trust, Exeter, Devon EX2 5DW, UK.

Received: 19 February 2019 Accepted: 25 June 2019

Published online: 03 July 2019

\section{References}

1. Reid BM, Permuth JB, Sellers TA. Epidemiology of ovarian cancer: a review. Cancer Biol Med. 2017;14:9-32.

2. Loizzi V, Del Vecchio V, Gargano G, De Liso M, Kardashi A, Naglieri E, Resta L, Cicinelli E, Cormio G. Biological pathways involved in tumor angiogenesis and bevacizumab based anti-angiogenic therapy with special references to ovarian cancer. Int J Mol Sci. 2017;18:1967.

3. Stone RL, Sood AK, Coleman RL. Collateral damage: toxic effects of targeted antiangiogenic therapies in ovarian cancer. Lancet Oncol. 2010;11:465-75.

4. Tateo S, Mereu L, Salamano S, Klersy C, Barone M, Spyropoulos AC, Piovella F. Ovarian cancer and venous thromboembolic risk. Gynecol Oncol. 2005;99:119-25.

5. Scappaticci FA, Skillings JR, Holden SN, Gerber HP, Miller K, Kabbinavar F, Bergsland E, Ngai J, Holmgren E, Wang J, Hurwitz H. Arterial thromboembolic events in patients with metastatic carcinoma treated with chemotherapy and bevacizumab. J Natl Cancer Inst. 2007;99:1232-9.
6. Perren TJ, Swart AM, Pfisterer J, Ledermann JA, Pujade-Lauraine E, Kristensen G, Carey MS, Beale P, Cervantes A, Kurzeder C, et al. A phase 3 trial of bevacizumab in ovarian cancer. N Engl J Med. 2011;365:2484-96.

7. Monk BJ, Minion LE, Coleman RL. Anti-angiogenic agents in ovarian cancer: past, present, and future. Ann Oncol. 2016;27(Suppl 1):i33-9.

8. Pranjol MZI, Gutowski NJ, Hannemann M, Whatmore JL. Cathepsin D nonproteolytically induces proliferation and migration in human omental microvascular endothelial cells via activation of the ERK1/2 and PI3K/AKT pathways. Biochim Biophys Acta Mol Cell Res. 2017;1865:25-33.

9. Winiarski BK, Wolanska KI, Rai S, Ahmed T, Acheson N, Gutowski NJ, Whatmore JL. Epithelial ovarian cancer-induced angiogenic phenotype of human omental microvascular endothelial cells may occur independently of VEGF signaling. Transl Oncol. 2013;6:703-14.

10. Pranjol MZ, Gutowski N, Hannemann M, Whatmore J. The potential role of the proteases cathepsin D and cathepsin L in the progression and metastasis of epithelial ovarian cancer. Biomolecules. 2015;5:3260-79.

11. Pranjol MZI, Gutowski NJ, Hannemann M, Whatmore JL. Cathepsin $L$ induces proangiogenic changes in human omental microvascular endothelial cells via activation of the ERK1/2 pathway. Curr Cancer Drug Targets. 2019;19:231-42.

12. Camby I, Le Mercier M, Lefranc F, Kiss R. Galectin-1: a small protein with major functions. Glycobiology. 2006;16:137R-57R.

13. Hsu YL, Wu CY, Hung JY, Lin YS, Huang MS, Kuo PL. Galectin-1 promotes lung cancer tumor metastasis by potentiating integrin alpha6beta4 and Notch1/Jagged2 signaling pathway. Carcinogenesis. 2013;34:1370-81.

14. van den Brule F, Califice S, Garnier F, Fernandez PL, Berchuck A, Castronovo V. Galectin-1 accumulation in the ovary carcinoma peritumoral stroma is induced by ovary carcinoma cells and affects both cancer cell proliferation and adhesion to laminin-1 and fibronectin. Lab Invest. 2003:83:377-86.

15. Chiariotti L, Berlingieri MT, Battaglia C, Benvenuto G, Martelli ML, Salvatore P, Chiappetta G, Bruni CB, Fusco A. Expression of galectin-1 in normal human thyroid gland and in differentiated and poorly differentiated thyroid tumors. Int J Cancer. 1995;64:171-5.

16. Ito K, Scott SA, Cutler S, Dong LF, Neuzil J, Blanchard H, Ralph SJ. Thiodigalactoside inhibits murine cancers by concurrently blocking effects of galectin-1 on immune dysregulation, angiogenesis and protection against oxidative stress. Angiogenesis. 2011;14:293-307.

17. van den Brule FA, Waltregny D, Castronovo V. Increased expression of galectin-1 in carcinoma-associated stroma predicts poor outcome in prostate carcinoma patients. J Pathol. 2001;193:80-7.

18. Li S, Xu HX, Wu CT, Wang WQ, Jin W, Gao HL, Li H, Zhang SR, Xu JZ, Qi ZH, et al. Angiogenesis in pancreatic cancer: current research status and clinical implications. Angiogenesis. 2018;22:15-36.

19. Chen L, Yao Y, Sun L, Zhou J, Liu J, Wang J, Li J, Tang J. Clinical implication of the serum galectin-1 expression in epithelial ovarian cancer patients. J Ovarian Res. 2015;8:78-88.

20. Zhang P, Zhang P, Shi B, Zhou M, Jiang H, Zhang H, Pan X, Gao H, Sun H, Li Z. Galectin-1 overexpression promotes progression and chemoresistance to cisplatin in epithelial ovarian cancer. Cell Death Dis. 2014;5:e991.

21. Thijssen VL, Postel R, Brandwijk RJ, Dings RP, Nesmelova I, Satijn S, Verhofstad N, Nakabeppu Y, Baum LG, Bakkers J, et al. Galectin-1 is essential in tumor angiogenesis and is a target for antiangiogenesis therapy. Proc Natl Acad Sci USA. 2006;103:15975-80

22. Croci DO, Salatino M, Rubinstein N, Cerliani JP, Cavallin LE, Leung HJ, Ouyang J, Ilarregui JM, Toscano MA, Domaica Cl, et al. Disrupting galectin-1 interactions with $\mathrm{N}$-glycans suppresses hypoxia-driven angiogenesis and tumorigenesis in Kaposi's sarcoma. J Exp Med. 2012;209:1985-2000.

23. D'Haene N, Sauvage S, Maris C, Adanja I, Le Mercier M, Decaestecker C, Baum L, Salmon I. VEGFR1 and VEGFR2 involvement in extracellular galectin-1- and galectin-3-induced angiogenesis. PLOS ONE. 2013;8:e67029.

24. Winiarski BK, Acheson N, Gutowski NJ, McHarg S, Whatmore JL. An improved and reliable method for isolation of microvascular endothelial cells from human omentum. Microcirculation. 2011;18:635-45.

25. Gilks CB. Pathology of serous tumours. In: Soslow RA, Tornos C, editors. Diagnostic pathology of ovarian tumors. 1st ed. New York: Springer; 2011. p. 55-73.

26. Pereira A, Perez-Medina T, Magrina JF, Magtibay PM, Rodriguez-Tapia A, Peregrin I, Mendizabal E, Ortiz-Quintana L. International federation of gynecology and obstetrics staging classification for cancer of the ovary, fallopian tube, and peritoneum: estimation of survival in patients 
with node-positive epithelial ovarian cancer. Int J Gynecol Cancer. 2015;25:49-54

27. Werner D, Battmann A, Steinmetz K, Jones T, Lamb T, Martinez M, Altmannsberger HM, Al-Batran SE. The validation of a novel method combining both HER2 immunohistochemistry and HER2 dual-colour silver in situ hybridization on one slide for gastric carcinoma testing. J Trans| Med. 2014;12:160

28. Weidner N. Current pathologic methods for measuring intratumoral microvessel density within breast carcinoma and other solid tumors. Breast Cancer Res Treat. 1995;36:169-80.

29. Kukreja I, Kapoor P, Deshmukh R, Kulkarni V. VEGF and CD 34: a correlation between tumor angiogenesis and microvessel density-an immunohistochemical study. J Oral Maxillofac Pathol. 2013;17:367-73.

30. Van Ry PM, Wuebbles RD, Key M, Burkin DJ. Galectin-1 protein therapy prevents pathology and improves muscle function in the $\mathrm{mdx}$ mouse model of Duchenne muscular dystrophy. Mol Ther. 2015;23:1285-97.

31. Thijssen VL, Barkan B, Shoji H, Aries IM, Mathieu V, Deltour L, Hackeng TM, Kiss R, Kloog Y, Poirier F, Griffioen AW. Tumor cells secrete galectin-1 to enhance endothelial cell activity. Cancer Res. 2010;70:6216-24.

32. Nickel W. Unconventional secretory routes: direct protein export across the plasma membrane of mammalian cells. Traffic. 2005;6:607-14.

33. Toscano MA, Campagna L, Molinero LL, Cerliani JP, Croci DO, llarregui JM, Fuertes MB, Nojek IM, Fededa JP, Zwirner NW, et al. Nuclear factor (NF)kappaB controls expression of the immunoregulatory glycan-binding protein galectin-1. Mol Immunol. 2011;48:1940-9.

34. Baum LG, Seilhamer JJ, Pang M, Levine WB, Beynon D, Berliner JA. Synthesis of an endogeneous lectin, galectin-1, by human endothelial cells is up-regulated by endothelial cell activation. Glycoconj J. 1995;12:63-8.

35. Guo Z, Huo J, Di J, Zeng S, Liu J, Xing F. PI3K pathway inhibitor LY294002 alters Jurkat T cell biobehaviours via ERK1/2-ICBP90 mediation. Cent Eur J Biol. 2014;9:739-48.

36. Hsieh SH, Ying NW, Wu MH, Chiang WF, Hsu CL, Wong TY, Jin YT, Hong TM, Chen YL. Galectin-1, a novel ligand of neuropilin-1, activates VEGFR-2 signaling and modulates the migration of vascular endothelial cells. Oncogene. 2008;27:3746-53.
37. Atlas THP. LGALS1 in adipose tissue. n.d.

38. Jung EJ, Moon HG, Cho Bl, Jeong CY, Joo YT, Lee YJ, Hong SC, Choi SK, Ha WS, Kim JW, et al. Galectin-1 expression in cancer-associated stromal cells correlates tumor invasiveness and tumor progression in breast cancer. Int J Cancer. 2007;120:2331-8.

39. Kim HJ, Jeon HK, Cho YJ, Park YA, Choi JJ, Do IG, Song SY, Lee YY, Choi CH, Kim TJ, et al. High galectin-1 expression correlates with poor prognosis and is involved in epithelial ovarian cancer proliferation and invasion. Eur J Cancer. 2012;48:1914-21.

40. Orozco CA, Martinez-Bosch N, Guerrero PE, Vinaixa J, Dalotto-Moreno T, Iglesias M, Moreno M, Djurec M, Poirier F, Gabius HJ, et al. Targeting galectin-1 inhibits pancreatic cancer progression by modulating tumor-stroma crosstalk. Proc Natl Acad Sci USA. 2018;115:E3769-78.

41. Winiarski BK, Cope N, Alexander M, Pilling LC, Warren S, Acheson N, Gutowski NJ, Whatmore JL. Clinical relevance of increased endothelia and mesothelial expression of proangiogenic proteases and VEGFA in the omentum of patients with metastatic ovarian high-grade serous carcinoma. Transl Oncol. 2014;7(267-276):e264.

42. Schulz H, Schmoeckel E, Kuhn C, Hofmann S, Mayr D, Mahner S, Jeschke U. Galectins-1, -3, and -7 are prognostic markers for survival of ovarian cancer patients. Int J Mol Sci. 2017;18:1230.

43. Griffioen AW, van der Schaft DW, Barendsz-Janson AF, Cox A, Struijker Boudier HA, Hillen HF, Mayo KH. Anginex, a designed peptide that inhibits angiogenesis. Biochem J. 2001;354:233-42.

44. Wu MH, Ying NW, Hong TM, Chiang WF, Lin YT, Chen YL. Galectin-1 induces vascular permeability through the neuropilin-1/vascular endothelial growth factor receptor-1 complex. Angiogenesis. 2014;17:839-49.

\section{Publisher's Note}

Springer Nature remains neutral with regard to jurisdictional claims in published maps and institutional affiliations.
Ready to submit your research? Choose BMC and benefit from:

- fast, convenient online submission

- thorough peer review by experienced researchers in your field

- rapid publication on acceptance

- support for research data, including large and complex data types

- gold Open Access which fosters wider collaboration and increased citations

- maximum visibility for your research: over $100 \mathrm{M}$ website views per year

At BMC, research is always in progress.

Learn more biomedcentral.com/submissions 